\title{
$\mathrm{N}$-杂环卡宾 Ru 配合物的合成及其在 Suzuki 偶联反应中的催化应用
}

\author{
李晓微周晋禚淑萍* \\ (山东理工大学化学工程学院 淄博 255049)
}

\begin{abstract}
摘要 合成了两个新的氮杂环卡宾金属钓配合物 $\mathbf{1}$ 和 $\mathbf{2}$, 通过核磁共振氢谱、核磁共振碳谱、红外光谱和元素分析对 其结构进行了表征，同时, $\mathrm{X}$ 射线单晶衍射确证了配合物 $\mathbf{2}$ 的结构为 $c i s(\mathrm{I})$ 顺式构型. 化合物 $\mathbf{1}$ 和 $\mathbf{2}$ 均能在温和的反应条 件下有效催化卤代芳烃和苯硼酸的 Suzuki 偶联反应, 并表现出较高的催化活性.
\end{abstract}

关键词 N-杂环卡宾; 钓配合物; 催化; Suzuki 偶联

\section{Synthesis of N-Heterocyclic Carbene Ruthenium Complex and Application as Catalyst for Suzuki Coupling Reaction}

\author{
Li, Xiaowei Zhou, Jin Zhuo, Shuping* \\ (School of Chemical Engineering, Shandong University of Technology, Zibo 255049)
}

\begin{abstract}
Two new complexes 1 and 2 were synthesized and characterized by ${ }^{1} \mathrm{H}$ NMR, ${ }^{13} \mathrm{C}$ NMR, IR spectra and elemental analysis, and the crystal structure of cis(I) configuration $\mathbf{2}$ has been also determined by X-ray crystal diffraction. Complexes $\mathbf{1}$ and $\mathbf{2}$ have been found to catalyze the Suzuki coupling reaction of aryl halides and phenylboronic acid with high catalytic activity under the mild reaction conditions.

Keywords N-heterocyclic carbene; ruthenium complex; catalysis; Suzuki coupling
\end{abstract}

Suzuki 偶联反应是构筑联芳烃类化合物最有效的 方法之一，在天然产物、复杂药物中间体和功能材料的 合成中发挥着非常重要的作用, 该反应能够在温和的条 件下进行，且具有反应选择性高、底物来源广、有机硼 酸无毒、对环境友好等优点而广受青睐. 在 Suzuki 反应 过程中, 催化剂的选择对偶联反应的活性和选择性起着 决定性的作用, 是研究 Suzuki 偶联反应的关键. 近年来, 以 $\mathrm{N}$-杂环卡宾( $\mathrm{N}$-heterocyclic carbenes, NHCs)为配体形 成的金属配合物作为催化剂, 催化 Suzuki 偶联反应已成 为有机化学和均相催化研究领域的热点.

NHCs 作为一类重要的支撑配体, 它的研究历史可 以追溯到 1968 年 Öfele ${ }^{[1]}$ 和 Wanzlick ${ }^{[2]}$ 对 NHC 过渡金属 配合物的报道, 然而在当时 NHCs 并未引起科学家们的 广泛注意, 直到 1991 年, Arduengo 等 ${ }^{[3]}$ 首次分离得到稳 定的游离 NHC 之后, NHCs 及其金属配合物的研究才得 到快速发展成为金属有机化学、配位化学和均相催化方 面的热点课题. 与传统的有机膦配体相比, NHCs 具有
很强的 $\sigma$ 给电子能力、易于制备、稳定性高、配位结构 多样化等独特的优点, 几乎能够与所有的过渡金属形成 稳定的配合物而在催化有机反应中表现出很高的活性, 因此，是一类极具潜在应用价值的催化剂，它将在新一 代金属有机催化剂中部分取代或完全取代有机膦配体.

到目前为止，关于 NHCs 金属配合物的合成与催化 有机反应已得到广泛的研究 ${ }^{[4 ~ 13]}$, 如 NHC-Ru 催化加氢 反应 ${ }^{[14]} 、$ NHC-Pd 催化 Heck $^{[15]}$ 和 Suzuki-Miyaura $\left.{ }^{[16]}\right]^{1}$ 碳碳偶联反应、NHC-Rh 催化乙烯和硅烷的氢硅化反

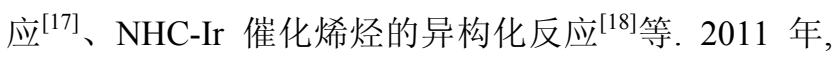
Chen 课题组 ${ }^{[19]}$ 曾报道了一系列吡啶功能化 $\mathrm{N}$-杂环卡宾 $\mathrm{Ru}$ 配合物并将其成功用于催化酮的转移加氢反应中, 然而, 吡啶功能化 N-杂环卡宾 Ru 配合物催化 Suzuki 偶 联反应的研究却相对较少, 在本工作中, 我们描述了两 个互为异构体的 $\mathrm{N}$-杂环卡宾 Ru 配合物，研究了 $\mathrm{N}$-杂环 卡宾前体盐抗衡离子和反应温度对配合物结构的影响, 该配合物在温和的反应条件下，能有效催化溴苯和苯硼

\footnotetext{
*E-mail: zhuosp_academic@163.com

Received April 6, 2014; revised May 11, 2014; published online June 9, 2014.

Project supported by the National Natural Science Foundation of China (No. 51302156).

国家自然科学基金(No. 51302156)资助项目.
} 
酸的 Suzuki 偶联反应. 本研究对开发新型高效 $N$-杂环 卡宾配位的金属配合物均相催化剂具有一定的意义.

\section{1 结果与讨论}

\section{$1.1 \mathrm{~N}$-杂环卡宾 Ru 配合物的合成与表征}

3-甲基-1-(2-吡啶基)碘化苯并咪唑鎓盐按文献[19] 方法制备, 其在碱三乙胺的作用下失去 2-位氢, 形成游 离的 3-甲基-1-(2-吡啶基)苯并咪唑 2-卡宾, 未经分离直 接与金属前体物 $\left[\mathrm{Ru}(\mathrm{CO})_{2} \mathrm{Cl}_{2}\right]_{n}$ 发生原位配位反应生成 $N$-杂环卡宾 $\mathrm{Ru}$ 配合物, 通过柱层析分离得到两种构型 的配合物 1 和 2 (Eq. 1), 其结构经 NMR, IR, 元素分析和 $\mathrm{X}$ 射线单晶衍射确定. 在配合物 1 和 2 的 ${ }^{1} \mathrm{H}$ NMR 谱中, 位于低场 $\delta 11$ 左右苯并咪唑盐2-位的质子信号消失, 表 明苯并咪唑盐已形成 $\mathrm{N}$-杂环卡宾且卡宾碳已与金属 $\mathrm{Ru}$ 配位, 这一结论被 ${ }^{13} \mathrm{C}$ NMR 谱中 $\delta 189.87$ 和 189.90 处 的信号进一步证实. 通过对比配合物 1 和 2 的 ${ }^{1} \mathrm{H}$ NMR 谱, 1 中吡啶 6-位氢的化学位移略小于 2 中吡啶 6-位氢 的化学位移, 参照相关文献 ${ }^{[20]}$, 我们推断出 $\mathbf{1}$ 为反式构 型 $\operatorname{trans}(\mathrm{I})$, 即与金属 $\mathrm{Ru}$ 配位的两个 I 互为反式, 而 2 为顺式构型 $c i s(\mathrm{I})$, 即与金属 $\mathrm{Ru}$ 配位的两个 I 互为顺式. 配合物 1 和 2 的红外光谱表明，位于 2060, 1992 和 2058, $1994 \mathrm{~cm}^{-1}$ 处的吸收带应归属于配合物 1 和 2 中两个羰 基的伸缩振动吸收峰. 通过分析苯并咪唑盐和反应条 件, 我们发现, 当苯并咪唑盐的抗衡离子为 $\mathrm{PF}_{6}^{-}$而非 $\mathrm{I}^{-}$ 时，反应在室温条件下仅得到一种顺式构型的配合物， 而在本文工作中, 苯并咪唑盐的抗衡离子为 $\mathrm{I}^{-}$, 反应在 加热回流的条件下, 金属前体 $\left[\mathrm{Ru}(\mathrm{CO})_{2} \mathrm{Cl}_{2}\right]_{n}$ 中的两个 $\mathrm{Cl}$ 被 I 取代形成六配位的 $N$-杂环卡宾羰基碘配合物，从而 得到两种构型的配合物, 由此可见, 咪唑盐的抗衡离子 和反应条件对配合物的结构有一定的影响.

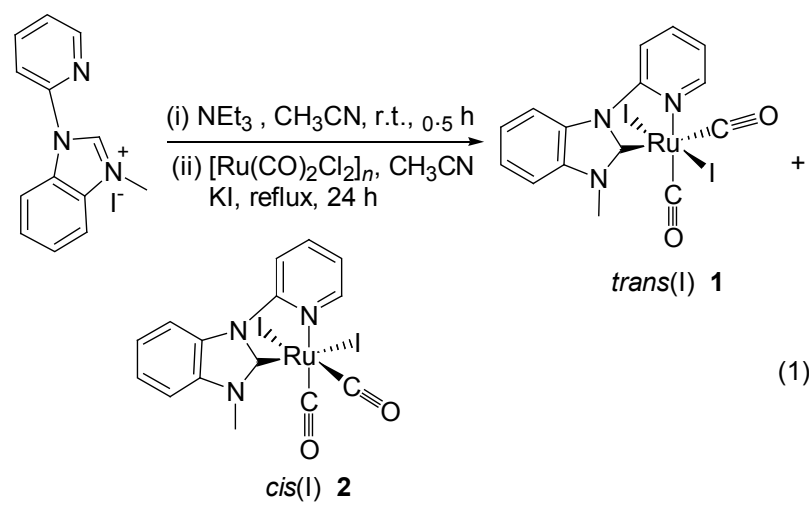

\section{2 晶体结构}

配合物 2 的晶体结构如图 1 所示, 其结构中, N-杂环 卡宾以双齿配位的模式与 $\mathrm{Ru}$ 配位, 金属 $\mathrm{Ru}$ 中心与来自 $N$-杂环卡宾配体的吡定氮、卡宾碳和两个 $\mathrm{CO}$ 、两个 I
配位形成扭曲的八面体构型，符合 $\mathrm{Ru}$ 配合物的配位方 式，其晶体结构中两个碘互为顺式，吡啶环与苯并咪唑 几乎共面. $\mathrm{Ru}$ 与卡宾碳键长 $\mathrm{Ru}(1)-\mathrm{C}(7)$ 为 2.000(6) $\AA$, 与吡啶氮键长 $\mathrm{Ru}-\mathrm{N}(3)$ 为 $2.147(4) \AA$, 这些数据均与文 献 [19] 报道的相似. 键角 $\mathrm{C}(15)-\mathrm{Ru}(1)-\mathrm{N}(3)$ 为 175.6(2) ${ }^{\circ}$, 说明 $\mathrm{C}(15), \mathrm{Ru}(1)$ 和 $\mathrm{N}(3)$ 原子是非线性的. 化合物 2 的部分键长和键角列于表 1 中.

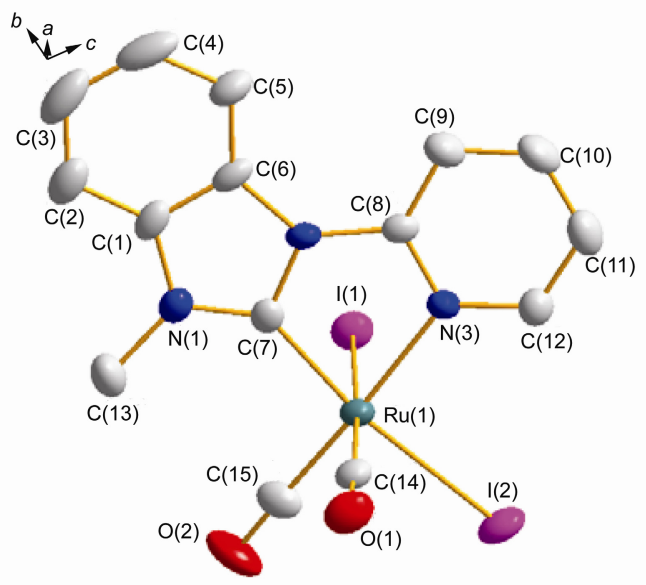

图 1 化合物 2 的分子结构

Figure 1 Molecular structure of complex 2

表 1 化合物 2 的部分键长 $(\AA)$ 和键角 $\left(^{\circ}\right)$

Table 1 Selected bond lengths $(\AA)$ and bond angles $\left({ }^{\circ}\right)$ of compound 2

\begin{tabular}{llll}
\hline $\mathrm{Ru}(1)-\mathrm{I}(1)$ & $2.7505(6)$ & $\mathrm{Ru}(1)-\mathrm{N}(3)$ & $2.147(4)$ \\
$\mathrm{Ru}(1)-\mathrm{I}(2)$ & $2.7674(6)$ & $\mathrm{Ru}(1)-\mathrm{C}(14)$ & $1.897(6)$ \\
$\mathrm{Ru}(1)-\mathrm{C}(7)$ & $2.000(6)$ & $\mathrm{Ru}(1)-\mathrm{C}(15)$ & $1.867(7)$ \\
$\mathrm{I}(1)-\mathrm{Ru}(1)-\mathrm{I}(2)$ & $92.353(18) \mathrm{N}(3)-\mathrm{Ru}(1)-\mathrm{C}(7)$ & $76.6(2)$ \\
$\mathrm{I}(1)-\mathrm{Ru}(1)-\mathrm{C}(7)$ & $85.84(13)$ & $\mathrm{I}(2)-\mathrm{Ru}(1)-\mathrm{C}(15)$ & $86.8(2)$ \\
$\mathrm{I}(2)-\mathrm{Ru}(1)-\mathrm{C}(7)$ & $171.28(17) \mathrm{N}(3)-\mathrm{Ru}(1)-\mathrm{C}(14)$ & $92.9(2)$ \\
$\mathrm{I}(1)-\mathrm{Ru}(1)-\mathrm{N}(3)$ & $90.96(11)$ & $\mathrm{N}(3)-\mathrm{Ru}(1)-\mathrm{C}(15)$ & $175.6(2)$ \\
$\mathrm{I}(2)-\mathrm{Ru}(1)-\mathrm{N}(3)$ & $94.93(12)$ & $\mathrm{C}(7)-\mathrm{Ru}(1)-\mathrm{C}(14)$ & $94.07(19)$ \\
$\mathrm{I}(1)-\mathrm{Ru}(1)-\mathrm{C}(14)$ & $176.0(2)$ & $\mathrm{C}(7)-\mathrm{Ru}(1)-\mathrm{C}(15)$ & $101.5(3)$ \\
$\mathrm{I}(2)-\mathrm{Ru}(1)-\mathrm{C}(14)$ & $88.33(14)$ & $\mathrm{C}(14)-\mathrm{Ru}(1)-\mathrm{C}(15)$ & $91.2(3)$ \\
$\mathrm{I}(1)-\mathrm{Ru}(1)-\mathrm{C}(15)$ & $84.92(18)$ & & \\
\hline
\end{tabular}

\section{$1.3 \mathrm{~N}$-杂环卡宾 Ru 配合物催化 Suzuki 偶联反应}

$\mathrm{N}$-杂环卡宾 $\mathrm{Ru}$ 配合物作为均相催化剂已被广泛用 于催化各类有机反应中，然而，异构化的 $\mathrm{N}$-杂环卡宾 $\mathrm{Ru}$ 配合物在催化 Suzuki 偶联反应中的研究却相对较少. 我们以溴苯和苯硼酸的 Suzuki 偶联反应为模型反应, N杂环卡宾 $\mathrm{Ru}$ 配合物为催化剂, 研究并对比了异构化的 $\mathrm{Ru}$ 配合物 1,2 和 $\operatorname{cis}(\mathrm{Cl})$ 在 Suzuki 偶联反应中的催化活 性(Eq. 2). 在 Suzuki 偶联反应中, 碱的种类是影响反应 性能的重要因素, 因此, 我们以偶联反应中常用的 1,4二氧六环为溶剂, 考察了不同的碱 $\mathrm{Cs}_{2} \mathrm{CO}_{3}, \mathrm{~K}_{2} \mathrm{CO}_{3}$, $\mathrm{KOH}, \mathrm{CH}_{3} \mathrm{COONa}, \mathrm{Na}_{2} \mathrm{CO}_{3}$ 在催化剂 1 用量为 $1 \mathrm{~mol} \%$ 时 
对反应性能的影响(表 2), 结果表明, 以 $\mathrm{K}_{2} \mathrm{CO}_{3}$ 为碱时, 偶联反应取得了最好的结果, $90{ }^{\circ} \mathrm{C}$ 反应 $4 \mathrm{~h}$, 溴苯几乎 能定量地发生反应, 溴苯的转化率达 $90 \%$, 而其他类型 碱没有 $\mathrm{K}_{2} \mathrm{CO}_{3}$ 效果理想.

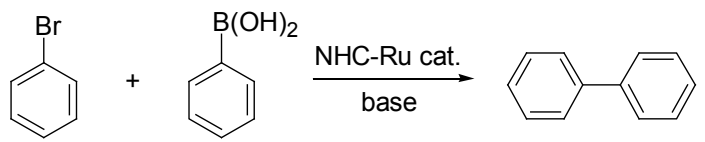

表 2 碱对 1 催化的 Suzuki 偶联反应的影响 ${ }^{a}$

Table 2 Effect of base on Suzuki coupling reaction catalyzed by 1

\begin{tabular}{clc}
\hline Entry & \multicolumn{1}{c}{ Base } & Conversion $^{b} \%$ \\
\hline 1 & $\mathrm{Cs}_{2} \mathrm{CO}_{3}$ & 51 \\
2 & $\mathrm{~K}_{2} \mathrm{CO}_{3}$ & 90 \\
3 & $\mathrm{KOH}$ & 80 \\
4 & $\mathrm{CH}_{3} \mathrm{COONa}$ & 20 \\
5 & $\mathrm{Na}_{2} \mathrm{CO}_{3}$ & 55 \\
\hline
\end{tabular}

${ }^{a}$ Experimental conditions: phenylboronic acid $(0.3 \mathrm{mmol})$, bromobenzene $(0.2$ mmol), $1 \mathrm{~mol} \%$ compound 1 , base $(0.6 \mathrm{mmol}), 1,4$-dioxane $(3 \mathrm{~mL}), 90{ }^{\circ} \mathrm{C}$ (oil bath), 4 h. ${ }^{b}$ The reaction was monitored by GC analysis.

为评价配合物 $\mathbf{1 , 2}$ 和 $\operatorname{cis}(\mathrm{Cl})$ 在 Suzuki 偶联反应中的 催化性能, 我们以 $1,4-$ 二氧六环为溶剂, $\mathrm{K}_{2} \mathrm{CO}_{3}$ 为碱, 在 氮气保护下油浴 $90{ }^{\circ} \mathrm{C}$ 反应, 考察以不同的配合物 $\mathbf{1 , 2}$ 和 $\operatorname{cis}(\mathrm{Cl})$ 做催化剂时溴苯和苯硼酸的反应情况(图 2), 从催化结果发现配合物 $\mathbf{1 , 2}$ 和 $\operatorname{cis}(\mathrm{Cl})$ 均能有效地催化溴 苯和苯硼酸的 Suzuki 偶联反应, 反应 $7 \mathrm{~h}$ 后, 溴苯几乎 能够完全转化, 转化率达 $96 \%$, 然而, 值得一提的是, 配合物 1 和 2 的催化活性略高于 $\operatorname{cis}(\mathrm{Cl})$, 这可能与配合 物结构中 $\mathrm{Ru}-\mathrm{I}$ 和 $\mathrm{Ru}-\mathrm{Cl}$ 的键长差异有关, 对于六配 位的 $\mathrm{N}$-杂环卡宾 $\mathrm{Ru}$ 配合物来说, 没有开放的活性位点 可供底物接近金属 $\mathrm{Ru}$ 中心，因而在催化反应过程中， 一个或两个辅助配体需断裂脱去, $\mathrm{Ru}-\mathrm{I}$ 键长 $[2.7505(6)$ 和 2.7674(6) $\AA]$ 较 $\mathrm{Ru}-\mathrm{Cl}$ 键长 $[2.4548(10)$ 和 2.4730(10) $\AA]$ 长, 因此 $\mathrm{Ru}-\mathrm{I}$ 键更容易断裂, 从而导致了较高的催 化活性. 配合物 $\mathbf{1}$ 较 $\mathbf{2}$ 的催化活性高, 这可能是由于构 型的差异所导致的.

为进一步研究 1 在 Suzuki 偶联反应中的催化性能, 我们用上述的反应条件, 以 1,4-二氧六环为溶剂, $\mathrm{K}_{2} \mathrm{CO}_{3}$ 为碱, $\mathrm{N}_{2}$ 保护下 $90{ }^{\circ} \mathrm{C}$ 反应 $10 \mathrm{~h}$, 考察其他含有不同取 代基的卤代芳烃与苯嗍酸的偶联反应，结果如表 3 所示， 发现以澳苯、对甲基溴苯、对乙酰基溴苯、对硝基溴苯 和碘苯为底物时反应进行的较好, 得到 $92 \%, 80 \%, 95 \%$, $98 \%, 99 \%$ 的产率(Entries 4 8), 而以氯苯、对甲基氯苯 和对乙酰基氯苯为底物时仅得到 24\%，18\%和 36\%的产 率(Entries 1 3). 对乙酰基氯苯(Entry 3)、对乙酰基澳苯 (Entry 6)和对硝基溴苯(Entry 7)与苯硼酸反应的分离收 率分别高于氯苯(Entry 1)、对甲基氯苯(Entry 2)和澳苯
(Entry 4)、对甲基溴苯(Entry 5), 从这些数据可以看出, 卤代芳烃结构中含有吸电子基团有利于 Suzuki 偶联反 应的进行.

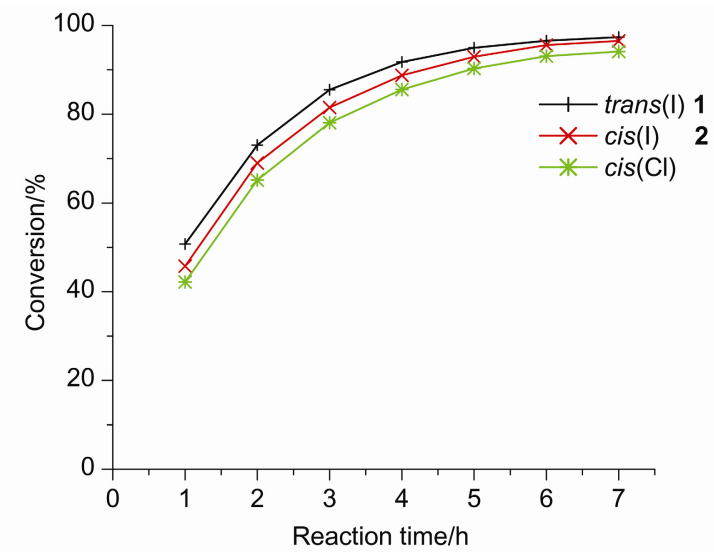

图 2 化合物 $\operatorname{trans}(\mathrm{I}) \mathbf{1}, \operatorname{cis}(\mathrm{I}) \mathbf{2}$ 和 $\operatorname{cis}(\mathrm{Cl})$ 催化溴苯和苯嗍酸的 Suzuki 偶联反应转化率一反应时间图

Figure 2 Plot of conversion vs reaction time for the Suzuki-Miyaura cross-coupling reaction of bromobenzene and phenylboronic acid with complexes $\operatorname{trans}(\mathrm{I}) \mathbf{1}, \operatorname{cis}(\mathrm{I}) \mathbf{2}$ and $\operatorname{cis}(\mathrm{Cl})^{[19]}$ as catalyst precursors

Experimental conditions: phenylboronic acid $(0.3 \mathrm{mmol})$, bromobenzene $(0.2 \mathrm{mmol}), 1 \mathrm{~mol} \%$ catalyst $\left[\operatorname{trans}(\mathrm{I}) \mathbf{1}, \operatorname{cis}(\mathrm{I}) 2\right.$ and $\left.\operatorname{cis}(\mathrm{Cl})^{[19]}\right], \mathrm{K}_{2} \mathrm{CO}_{3}$ (0.6 mmol), 1,4-dioxane $(3 \mathrm{~mL})$ at $90{ }^{\circ} \mathrm{C}$ (oil bath). All reactions were monitored by GC analysis at 1, 2, 3, 4, 5, 6 and $7 \mathrm{~h}$ to detect the conversion instantly.

表 3 化合物 1 催化卤代芳烃与苯嗍酸的偶联反应 ${ }^{a}$

Table 3 Compound 1-catalyzed Suzuki coupling reaction of aryl halides and phenylboronic acid

Entry Phenylboronic acid

${ }^{a}$ Experimental conditions: phenylboronic acid $(0.3 \mathrm{mmol})$, bromobenzene $(0.2$ mmol), $1 \mathrm{~mol} \%$ compound $1, \mathrm{~K}_{2} \mathrm{CO}_{3}(0.6 \mathrm{mmol}), 1,4$-dioxane $(3 \mathrm{~mL}), 90{ }^{\circ} \mathrm{C}$ (oil bath), $10 \mathrm{~h}$. 


\section{2 结论}

通过改变 $\mathrm{N}$-杂环卡宾前体盐抗衡离子、优化反应条 件合成了两个 $\mathrm{N}$-杂环卡宾 Ru 化合物, 利用核磁共振氢 谱、核磁共振碳谱、红外光谱和元素分析对化合物进行 了表征，同时，利用 $\mathrm{X}$ 射线单晶衍射证实了化合物 $\mathbf{2}$ 的 分子结构. 研究和对比了化合物 $\mathbf{1}, \mathbf{2}$ 以及 $\operatorname{cis}(\mathrm{Cl})$ 在溴苯 与苯硼酸的 Suzuki 偶联反应中的催化性能, 结果表明, 化合物 $\mathbf{1}$ 和 $\mathbf{2}$ 的催化活性高于 $\operatorname{cis}(\mathrm{Cl})$, 且取代基的电子 效应对偶联反应的产率有重要的影响.

\section{3 实验部分}

\section{1 仪器与试剂}

Bruker AM-500 型核磁共振仪 $\left(\mathrm{CDCl}_{3}\right.$ 或 DMSO- $d_{6}$ 为溶剂, TMS 为内标); Perkin-Elmer 240C 型元素分析仪; Nicolet NEXUS870 型红外光谱仪; 岛津 2010 气相色谱 仪. 所有反应均在干燥的 $\mathrm{N}_{2}$ 气氛下, Schlenk 瓶中进行. 实验所用试剂均为市售分析纯, 溶剂乙腈按照标准方法 经氢化钙回流后蒸出, 其他试剂和溶剂使用前未经纯 化, 直接使用. 柱层析使用 $300 \sim 400$ 目硅胶. 卡宾前体 盐 3-甲基-1-(2-吡啶基)碘化苯并咪唑鎓盐 ${ }^{[21]}$ 和金属前体 物 $\left[\mathrm{Ru}(\mathrm{CO})_{2} \mathrm{Cl}_{2}\right]_{n}{ }^{[22]}$ 按文献方法合成.

\section{2 配合物的合成}

无水无氧条件下, 将 3-甲基-1-(2-吡啶基)碘化苯并 咪唑盐 $(0.34 \mathrm{~g}, 1.0 \mathrm{mmol})$ 加入到 $100 \mathrm{~mL}$ 的 Schlenk 瓶中, 然后向其中加入 $50 \mathrm{~mL}$ 乙腈和三乙胺 $(0.54 \mathrm{~mL}, 4.0$ $\mathrm{mmol}$ ), 室温摚拌反应 $0.5 \mathrm{~h}$ 后, 向反应体系中加入 $\left[\mathrm{Ru}(\mathrm{CO})_{2} \mathrm{Cl}_{2}\right]_{n}(0.23 \mathrm{~g}, 1.0 \mathrm{mmol})$ 和过量的碘化钾, 反应 混合物加热回流 $24 \mathrm{~h}$. 反应完毕后, 过滤, 减压除去溶 剂, 粗产品用硅胶色谱柱分离, 先用二氯甲烷洗脱得到 黄色固体 $1(0.19 \mathrm{~g})$, 产率为 30\%. 待 $\mathbf{1}$ 收集完后, 用二 氯甲烷一丙酮 $(V: V=20: 1)$ 洗脱得到浅黄色固体 2 $(0.13 \mathrm{~g})$, 产率为 $21 \%$. 相关数据如下:

反式二碘-二羰基-[3-甲基-1-(2-吡啶基)苯并咪唑] 合钓(1): ${ }^{1} \mathrm{H}$ NMR (500 MHz, DMSO- $d_{6}$ ) $\delta: 9.41$ (d, $J=5.5$ $\mathrm{Hz}, 1 \mathrm{H}, 6-\mathrm{H}$ of Py), 8.65 (d, $J=8 \mathrm{~Hz}, 1 \mathrm{H}, \mathrm{ArH}), 8.50$ (d, $J=7.0 \mathrm{~Hz}, 1 \mathrm{H}, \mathrm{ArH}), 8.43$ (t, $J=8.5 \mathrm{~Hz}, 1 \mathrm{H}, 5-\mathrm{H}$ of Py), 8.00 (d, $J=8.5 \mathrm{~Hz}, 1 \mathrm{H}, 3-\mathrm{H}$ of Py), 7.77 (t, $J=6.5 \mathrm{~Hz}, 1 \mathrm{H}$, 4-H of Py), 7.64 7.62 (m, 2H, $\mathrm{ArH}), 4.21\left(\mathrm{~s}, 3 \mathrm{H}, \mathrm{CH}_{3}\right)$; ${ }^{13} \mathrm{C}$ NMR (DMSO- $\left.d_{6}, 125 \mathrm{MHz}\right) \delta: 198.99,193.76$, $189.87,154.45,152.90,143.18,135.97,130.65,125.97$, $125.79 ， 123.52,114.34,113.36,112.77,35.60$; FT-IR (KBr) $v$ : 3070, 3066, 3020, 2994, 2880, 2060, 1992, 1617, 1579, 1477, 1449, 1417, 1270, 1215, 989, $727 \mathrm{~cm}^{-1}$. Anal. calcd for $\mathrm{C}_{15} \mathrm{H}_{11} \mathrm{I}_{2} \mathrm{~N}_{3} \mathrm{O}_{2} \mathrm{Ru}$ : C 29.03, H 1.77, N 6.77; found
C 28.83, H 1.92, N 6.88.

顺式二碘-二羰基-[3-甲基-1-(2-吡啶基)苯并咪唑] 合钓 (2): ${ }^{1} \mathrm{H}$ NMR (500 MHz, DMSO- $\left.d_{6}\right) \delta: 9.78(\mathrm{~d}, J=5$ $\mathrm{Hz}, 1 \mathrm{H}, 6-\mathrm{H}$ of Py), 8.68 (d, $J=8.0 \mathrm{~Hz}, 1 \mathrm{H}, \mathrm{ArH}), 8.52$ (d, $J=8.0 \mathrm{~Hz}, 1 \mathrm{H}, \mathrm{ArH}), 8.41(\mathrm{t}, J=8.5 \mathrm{~Hz}, 1 \mathrm{H}, 5-\mathrm{H}$ of Py), $8.02(\mathrm{~d}, J=8 \mathrm{~Hz}, 1 \mathrm{H}, 3-\mathrm{H}$ of Py), 7.69 (t, $J=6.5 \mathrm{~Hz}, 1 \mathrm{H}$, 4-H of Py), 7.65 7.62 (m, 2H, ArH), 4.20 (s, 3H, $\mathrm{CH}_{3}$ ); ${ }^{13} \mathrm{C}$ NMR (DMSO- $\left.d_{6}, 125 \mathrm{MHz}\right) \delta: 198.98,193.75$, $189.90,154.44,152.89,143.18,135.96,130.64,125.79$, 123.52, 114.33, 113.35, 112.77, 35.60; FT-IR (KBr) v: 3067, 3060, 3019, .2990, 2872, 2058, 1994, 1624, 1560, $1482,1450,1420,1268,1220,986,721 \mathrm{~cm}^{-1}$. Anal. calcd for $\mathrm{C}_{15} \mathrm{H}_{11} \mathrm{I}_{2} \mathrm{~N}_{3} \mathrm{O}_{2} \mathrm{Ru}$ : C 29.03, H 1.77, N 6.77; found $\mathrm{C}$ 28.90, H 1.89, N 6.85 .

\section{3 晶体结构的测定}

单晶 $\mathrm{X}$ 射线衍射测定配合物 $\mathbf{2}$ 的晶体结构，挑选尺 寸适当 $(0.28 \mathrm{~mm} \times 0.24 \mathrm{~mm} \times 0.22 \mathrm{~mm})$ 的晶体, 放于 Bruker SMART CCD 单晶衍射仪上, 采用石墨单色化的 Mo $\mathrm{K} \alpha$ 射线 $(\lambda=0.71073 \AA)$ 收集数据. 晶体结构采用 SHELXS-97 ${ }^{[23]}$ 程序对 $F^{2}$ 进行精修, 对非氢原子和各向 异性参数进行修正, 相关晶体学数据存于英国剑桥晶体 数据中心, CCDC 928615, 可通过 http://www.ccdc.cam. ac.uk/conts/retrieving.html 或 e-mail: deposit@ccdc.cam. ac.uk 免费获取, 晶体结构精修参数见辅助材料.

\subsection{Suzuki 偶联反应}

在 $\mathrm{N}_{2}$ 置换过的干燥的 Schlenk 管中加入 $\mathrm{N}$-杂环卡 宾 $\mathrm{Ru}$ 配合物 $(0.01 \mathrm{~mol})$, 碳酸钾 $(0.6 \mathrm{mmol})$ 和苯硼酸 $(0.3 \mathrm{mmol}), \mathrm{N}_{2}$ 切换并保护, 然后加入卤代芳烃 $(0.2$ $\mathrm{mmol})$ 和 1,4 -二氧六环 $(3 \mathrm{~mL})$, 在 $90{ }^{\circ} \mathrm{C}$ 搅拌反应, $\mathrm{GC}$ 跟 踪. 反应结束后, 冷却至室温, 过滤, 减压除去溶剂后, 柱层析分离纯化得到偶联产物.

辅助材料(Supporting Information) 化合物 $\mathbf{2}$ 的晶体结 构精修参数以及化合物 $\mathbf{1}$ 和 2 的 ${ }^{1} \mathrm{H} N \mathrm{NMR}$ 和 ${ }^{13} \mathrm{C}$ NMR. 这 些材料可以免费从本刊网站(http://sioc-journal.cn/)上下 载.

\section{References}

[1] Öfele, K. J. J. Organomet. Chem. 1968, 12, 42.

[2] Wanzlick, H. W.; Schönherr, H. J. Angew. Chem., Int. Ed. Engl. 1968, 7, 141 .

[3] Arduengo, A. J.; Harlow, R. L.; Kilne, M. J. Am. Chem. Soc. 1991, 113,361 .

[4] Kolychev, E. L.; Kronig, S.; Brandhorst, K.; Freytag, M.; Jones, P. G.; Tamm, M. J. Am. Chem. Soc. 2013, 135, 12448.

[5] Prokofjevs, A.; Boussonnière, A.; Li, L. F.; Bonin, H.; Lacôte, E.; Curran, D. P.; Vedejs, E. J. Am. Chem. Soc. 2012, 134, 12281. 
[6] Li, J. Y.; Peng, J. J.; Li, X. N.; Ma, L.; Bai, Y.; Zhang, G. D.; Lai, G. Q. Chin.J. Org. Chem. 2010, 30, 1468 (in Chinese). (厉嘉云, 彭家建, 李小年, 马否, 白赢, 张国栋, 来国桥, 有机 化学, 2010, 30, 1468.)

[7] Giuseppe, A. D.; Castarlenas, R.; Pérez-Torrente, J. J.; Crucianelli, M.; Polo, V.; Sancho, R.; Lahoz, F. J.; Oro, L. A. J. Am. Chem. Soc. 2012, 134, 8171.

[8] Ingleson, M. J.; Layfield, R. A. Chem. Commun. 2012, 48, 3579.

[9] Yu, H. W.; Tong, Q. S.; Jia, L.; Jin, Z. L.; Shi, J. C. Chin. J. Org. Chem. 2011, 31, 742 (in Chinese).

(于宏伟, 童庆松, 贾莉, 金子林, 施继成, 有机化学, 2011, 31, 742.)

[10] Hashmi, A. S. K.; Yu, Y.; Rominger, F. Organometallics 2012, 31, 895.

[11] Zhang, W. X.; Zhang X. Q.; Luo, M. M. Chin. J. Chem. 2012, 30, 1423 .

[12] Zhang, R.; Wang, D.; Xu, Q.; Jiang, J. J.; Shi, M. Chin. J. Chem. 2012, 30, 1295.

[13] Zheng, S. Z.; Peng, X. G.; Liu, J. M.; Sun, W.; Xia, C. G. Chin. J. Chem. 2007, 25, 1065.
[14] Valente, C.; Calimsiz, S.; Hoi, K. H.; Mallik, D.; Sayah, M.; Organ, M. G. Angew. Chem., Int. Ed. 2012, 51, 3314.

[15] Lin, Y. C.; Hsueh, H. H.; Kanne, S.; Chang, L. K.; Liu, F. C.; Lin, I. J. B. Organometallics 2013, 32, 3859.

[16] Yuan, D.; Huynh, H. V. Organometallics 2010, 29, 6020.

[17] Wu, Y.; Karttunen, V. A.; Parker, S.; Genest, A.; Rösch, N. Organometallics 2013, 32, 2363.

[18] Chianese, A. R.; Shaner, S. E.; Tendler, J. A.; Pudalov, D. M.; Shopov, D. Y.; Kim, D.; Rogers, S. L.; Mo, A. Organometallics 2012, 31, 7359.

[19] Li, X. W.; Wang, G. F.; Chen, F.; Li, Y. Z.; Chen, X. T.; Xue, Z. L. Inorg. Chim. Acta 2011, 378, 280.

[20] Cheng, Y.; Xu, H. J.; Sun, J. F.; Li, Y. Z.; Chen, X. T.; Xue, Z. L. Dalton Trans. 2009, 35, 7132.

[21] Chianese, A. R.; Bremer, P. T.; Wong, C.; Reynes, R. J. Organometallics 2009, 28, 5244.

[22] Mulhern, D.; Lan, Y. H.; Brooker, S.; Gallagher, J. F.; Gorls, H.; Rau, S.; Vos, J. G. Inorg. Chim. Acta 2006, 359, 736.

[23] Sheldrick, G. M. SHELXS-97 and SHELXL-97, Programs for Crystal Structure Refinement, University of Göttingen, Germany, 1997.

(Zhao, C.) 


\title{
辅助材料(Supporting Information)
}

\section{$\mathrm{N}$-杂环卡宾 Ru 配合物的合成及其在 Suzuki 偶联反应中的催化应用}

\author{
李晓微周 晋禚淑萍* \\ (山东理工大学化学工程学院 淄博 255049)
}

图 S1 化合物 $\mathbf{1}$ 的 ${ }^{1} \mathrm{H} \mathrm{NMR}$

Figure S1 ${ }^{1} \mathrm{H}$ NMR of complex 1

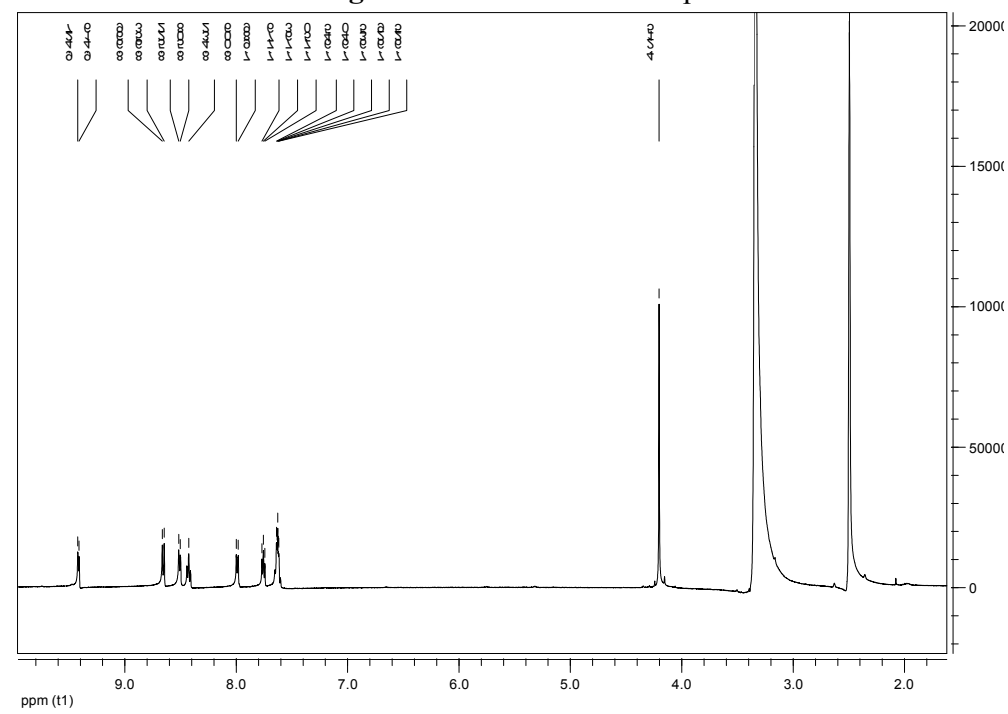

图 S2 化合物 2 的 ${ }^{1} \mathrm{H}$ NMR

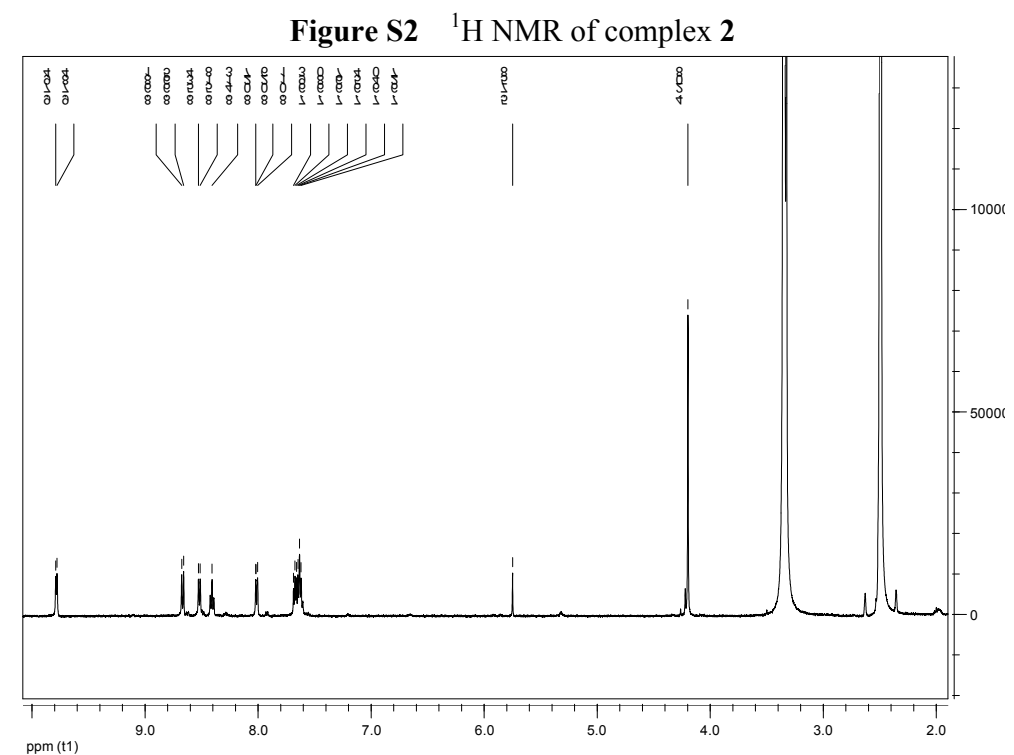


图 S3 化合物 1 的 ${ }^{13} \mathrm{C} \mathrm{NMR}$

Figure S3 ${ }^{13} \mathrm{C}$ NMR of complex 1

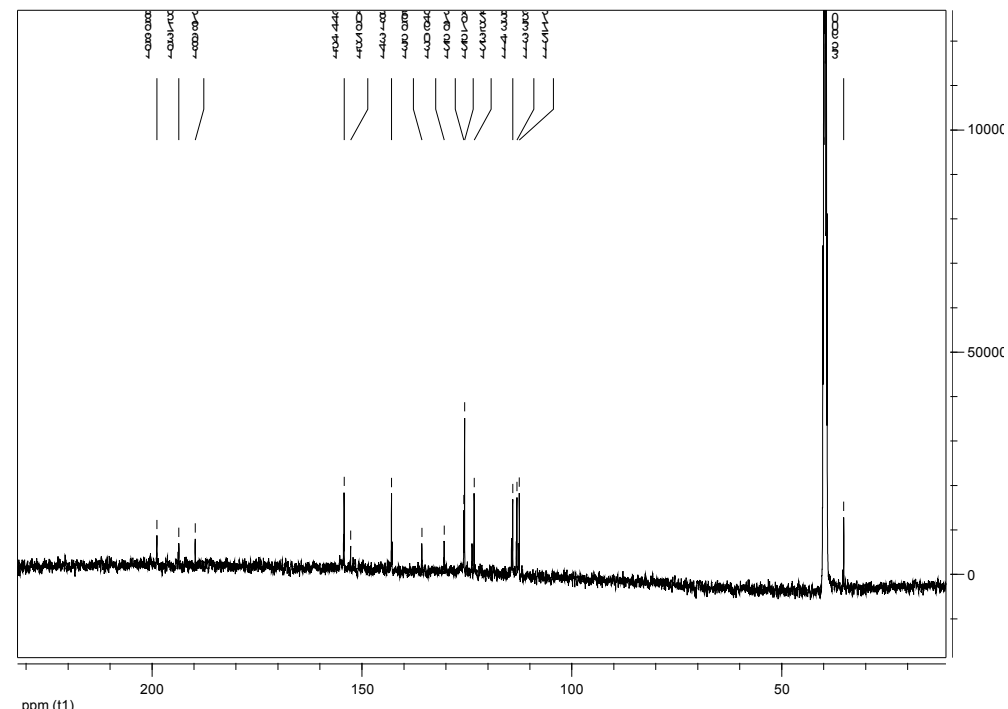

ppm (t1)

图 S4 化合物 2 的 ${ }^{13} \mathrm{C} \mathrm{NMR}$

Figure S4 ${ }^{13} \mathrm{C}$ NMR of complex 2

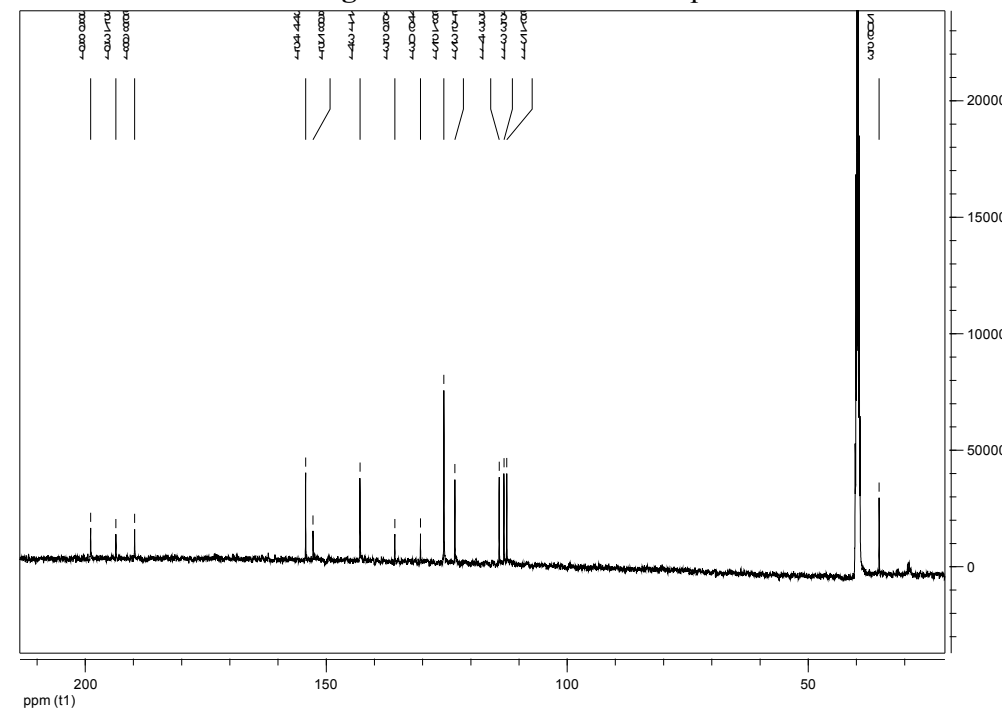


表 S1 化合物 2 的晶体学结构精修数据

Table S1 Crystal data and structural refinements for complex 2

\begin{tabular}{|c|c|}
\hline Complex & 2 \\
\hline Empirical formula & $\mathrm{C}_{15} \mathrm{H}_{11} \mathrm{I}_{2} \mathrm{~N}_{3} \mathrm{O}_{2} \mathrm{Ru}$ \\
\hline Formula weigh & 620.14 \\
\hline Crystal system & Orthorhombic \\
\hline Space group & Pna21 \\
\hline$a, \AA$ & $12.5770(14)$ \\
\hline$b, \AA$ & $12.9614(14)$ \\
\hline$c, \AA$ & $19.505(12)$ \\
\hline$\alpha\left(^{\circ}\right)$ & 90 \\
\hline$\beta\left(^{\circ}\right)$ & 90 \\
\hline$\gamma\left({ }^{\circ}\right)$ & 90 \\
\hline Volume, $\AA^{3}$ & $1785.1(3)$ \\
\hline$Z$ & 4 \\
\hline$\rho_{\text {calcd }}, \mathrm{g} / \mathrm{cm}^{3}$ & 2.307 \\
\hline$\mu, \mathrm{mm}^{-1}$ & 4.348 \\
\hline$F(000)$ & 1152 \\
\hline$\theta$ range, deg & 2.26 to 26.00 \\
\hline Limiting indices $h, k, l$ & $-14 \leqslant h \leqslant 15,-15 \leqslant k \leqslant 14,-11 \leqslant l \leqslant 13$ \\
\hline Reflections collected & 9137 \\
\hline Independent reflections $\left(R_{\text {int }}\right)$ & $3287(0.0502)$ \\
\hline Reflections with $I>2 \sigma(I)$ & 2925 \\
\hline Number of parameters & 209 \\
\hline Goodness-of-fit & 0.961 \\
\hline$R_{1}, w R_{2}(I>2 \sigma(I))$ & $R_{1}=0.0261, w R_{2}=0.0561$ \\
\hline$R_{1}, w R_{2}$ (all data) & $R_{1}=0.0308, w R_{2}=0.0573$ \\
\hline$\Delta \rho_{\min } / \Delta \rho_{\max }, e \cdot \AA^{-3}$ & $-0.575 / 0.513$ \\
\hline
\end{tabular}

